\title{
A Search for Short-Lived Transient Phenomenon in LS I+61 303
}

\author{
C. R. Shrader ${ }^{*} \&$ S. Sturner \\ Astrophysics Science Division, NASA Goddard Space Flight Center, Code 661, Greenbelt MD, 20771 \\ * also with Universities Space Research Association
}

\begin{abstract}
Motivated by reports of transient behavior on the circumstellar disk of the gamma-ray bright, X-ray binary system LSI +61303 we have undertaken a search for possible ionizing events. We have utilized the hard-X-ray monitoring database provided by the INTEGRAL Galactic Plane survey program.
\end{abstract}

Keywords: X-ray binaries - LS I+61303 - neutron stars - gamma rays.

PACS: $97.80 . \mathrm{Jp}$

\section{INTRODUCTION}

The galactic X-ray binary LS I+61 303 is one of three known gamma-ray emitting X-ray binaries. It consists of a Be primary, with a compact companion of unknown type, although it is likely a neutron star. It is a $\sim 50-200 \mathrm{mJy}$ Ghz radio source, modulated at the orbital period. The X-ray emission is also modulated at the orbital frequency, but both the radio/X-ray peaks are delayed by a significant fraction of the orbital period $(0.5 / 0.25)$ relative to periastron. Monitoring of the $\mathrm{H}$-alpha emission of the source, which is widely believed to trace the size of circumstellar Be star disks indicates periodic modulation, as well as episodic behavior. In particular, the disk appears to have either completely dissipated on < day timescales, or become ionized by some transient event[1]. The latter explanation is favored, as the timescale for the former seems unreasonably short. However, the $2-10-\mathrm{keV}$ intensity from the RXTE/ASM does not reveal any significant variation over that time interval. One possibility could be a higher energy transient event leading to ionization of the disk. In any case, the size and thermo-dynamical configuration of the circumstellar environment are of critical importance any models for the gamma-ray emission. We have thus undertaken a search of the hard-X-ray databases of INTEGRAL and Swift to search for transient emission from the direction of LSI +61303 . We report on the results of this analysis, and its implications on our understanding of the physics underlying LS I+61 303 and of gamma radiation from X-ray binaries in general.

\section{LS I+61 303: Overview of Basic Properties}

This Be X-ray binary ( $\mathrm{BO}$ Ve) has a 26.5-day orbital period and is characterized as a very low luminosity X-ray source $-5 \times 10^{33} \mathrm{ergs} / \mathrm{cm} 2 / \mathrm{s}(\mathrm{d} / 2 \mathrm{kpc})^{2}[1,2,3]$. This is consistent with the high-end of the luminosity distribution of coronal emission from Be stars, and some 4 orders of magnitude below typical XRBs or high-state Be transient events. It is likely a wind accretor, otherwise, is characterized by an extremely radiatively inefficient diskOrbit modulated as well as episodic radio emission has been reported, and it is an $\sim 100 \mathrm{MeV}-\mathrm{TeV} \gamma$-ray source. $\mathrm{X}$-ray and radio LC peaks are respectively $0.25,0.4$ phase past periastron. Its mass function requires inc $<-25^{\circ}$ for the secondary to be a $\mathrm{BH}$, and it is further noted that all known Be XRBs have neutron star secondaries.

\section{Model Scenarios}

Embedded Pulsar: In this model a fast-spinning pulsar is embedded in the dense circumstellar environment in systems such as LSI +61303 \& LS 50309[4]. A spin-powered magnetic propeller effect inhibits accretion. The radio 
pulsations are smeared out by scattering in the high density NS orbital environment. Similar to periastron passage in PSR 1259-63. The pulsar - OB star wind interactions are responsible for radio- $\gamma$ emission in this class of models. The main difficulty is the lack of discernable pulsations at any wavelength.
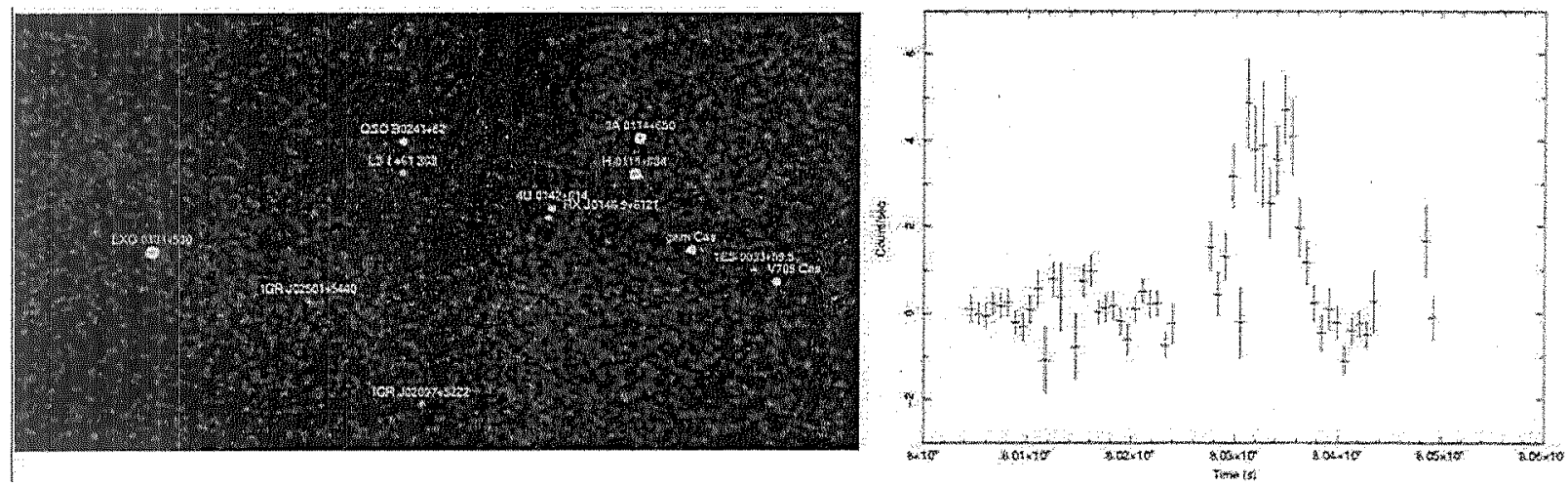

FIGURE 1. LHS: $20-40 \mathrm{keV}$ map of the Cassiopeia region, indicating the clear detection of LSI +61 303. RHS: apparent flaring event in the $20-40 \mathrm{keV}$ source intensity. The duration is nominally consistent with the duration of the apparent disk-ionizing event reported by [1], and the peak luminosity is $-10^{35.5} \mathrm{ergs} / \mathrm{s}$. Caution must be exercised however, as noise spikes of similar amplitude are seen in some contemporaneous frames. Searches of the Swift database have not yet revealed similar features.

Disk Jet Systems: These models are motivated by the quasar analogy[5]. The jet kinetic power in XRBS may be comparable to $L_{\text {edd }}$ of the binary systems (e.g. $\sim 10^{39} \mathrm{ergs} / \mathrm{s}$ for SS 433 ), and shocks can be triggered, accelerating $\mathrm{e}^{-}$ to $\sim \mathrm{TeV}$ energies. Each known $\gamma$-ray XRB is a variable radio sources and are pulse- or orbit-modulated X-ray sources. Problems remain: for example, disk accretion must have very low radiative efficiency, or material is expelled. Perhaps uniquely high jet-to-disk power systems are associated with sufficiently low $\tau \gamma$ environments. Another issue is that canonical X-ray novae such as GRO 1655-40 \& GRS 1915+105 exhibit large amplitude, episodic variations as well as short timescale, stochastic \& quasi-periodic variability, and $L x$ can approach $L_{\text {edd }}$.

Wind Accretion \& NS Magnetosphere: In this scenario density irregularities in the OB circumstellar environment interact with high-energy protons trapped in the pulsar magnetosphere.

\section{Possible Clues: Circumstellar Environment}

Be systems consist of dense circumstellar disks which interact with the compact secondaries. There can also be winds which are complex, consisting of plolar as well as equatorial components, and which are highly variable in intensity. This leads to transient outbursts in highly eccentric systems. Near-circular orbit systems will also vary with environment effects The size and density of the circumstellar disk is a determinant factor in variability amplitude, and the $H \alpha E W$ is a traces that environmemt. We examined a large number of INTEGRAL/IBIS images of the Cas region to look for short-timescale (ie $\sim$ days). We identified one intriguing event depicted in Fig 1 . The $20-50-\mathrm{keV}$ flux increased by a factor of $\sim 5-10$ for $\sim 1$ day. The mean luminosity during the event is $\sim 10^{35} \mathrm{ergs} / \mathrm{cm} 2 / \mathrm{s}$. This is reminiscent of the "fast transient" hard-X-ray selected sources recently categorized by INTEGRAL which are believed to involve irregular wind-powered accretion in dense circumstellar environments.

Finally, We would note the rather striking similarity between the X-ray and properties, and the primaries of LSI +61303 and Gamma Cas (and also HD110432). In each case, the X-ray luminsoties are a few $10^{33}$, the light curves are exceptionally flat (at least within the context of $\mathrm{Be} \mathrm{X}$-ray binaries), and there are no discernable pulsations. The difference is the radio emission, which is absent or orders of magnitude lower in Gamma Cas.

\section{REFERENCES}

1. Grundstrom, E.D., et al., 2006, astro-ph/0610608

2. Leahy, D., et al., ApJ, $1997,475,823$

3. Tavani, M., et al., 1998, ApJ, 497, L89

4. Dubus, G., 2006, astro-ph/0608262

5. Dermer, C.D., Boettcher, M., 2006, ApJ, 643, 1081

6. Aharonian, F., et al., 2006, A\&A, 460, 743 